\title{
Hemoglobin level significantly impacts the tumor cell survival fraction in humans after internal radiotherapy
}

\author{
Stephan Walrand ${ }^{1 *}$, Renaud Lhommel ${ }^{1}$, Pierre Goffette ${ }^{2}$, Marc Van den Eynde ${ }^{3}$, Stanislas Pauwels ${ }^{1}$ and \\ François Jamar ${ }^{1}$
}

\begin{abstract}
Background: Anemia is usually not taken into account in internal radiotherapy. We investigated whether the hemoglobin $(\mathrm{Hb})$ level could have an impact on the tumor response, as observed in external beam radiotherapy (EBRT).

Methods: Absorbed doses of 25 hepatic metastatic sites in eight patients who underwent a liver selective internal radiotherapy (SIRT) were computed by a 3D convolution of a dose deposition kernel with the ${ }^{90} \mathrm{Y}$ time-of-flight positron emission tomography (TOF-PET) images acquired following therapy. Early tumor response was assessed by comparing a follow-up FDG TOF-PET scan with a baseline scan. Hb level was measured on the day of the SIRT procedure.

Results: All patients displayed early tumor response increasing with the tumor-absorbed dose. Significant differences between patients were noted, the response slope correlating with the Hb level. After applying a global fit on all metastases using a tumor radiosensitivity modulated by a $\mathrm{Hb}$ enhancement factor (HEF) linearly dependent on the $\mathrm{Hb}$ level, a strong correlation $(R=0.96)$ was observed between the early response and the absorbed dose. Hb level had a major impact on tumor response by modulating HEF by a factor 6 .

Conclusions: These results prove the significant impact of $\mathrm{Hb}$ level on the tumor response and support the study of methods for correcting tumor hypoxia, such as intensively performed in EBRT. The quantitative analysis of the relationship between tumor doses and early response has the power to allow fast screening of such correction methods in limited patient series. Internal radiotherapy could be more efficient if performed earlier in the therapy line, when the disease- and treatment-related anemia remains limited.
\end{abstract}

Keywords: Liver SIRT, Internal radiotherapy, Dosimetry, Hemoglobin, Anemia, Dose-response.

\section{Background}

The importance of anemia as a prognostic factor of patient outcome in external beam radiotherapy (EBRT) of solid tumors has been documented in large clinical series and thoroughly reviewed [1-8]. This impact results from three related key points: (1) the hemoglobin $(\mathrm{Hb})$ level was shown to strongly correlate with tumor oxygenation in numerous cancers [9], (2) substantial data show that tumor hypoxia is involved in processes conferring a growth advantage and the development of a more malignant phenotype [10-15], and (3) hypoxic tumors are

\footnotetext{
* Correspondence: stephan.walrand@uclouvain.be

'Department of Nuclear Medicine, Cliniques Universitaires Saint Luc, Université Catholique de Louvain, Avenue Hippocrate 10, Brussels 1200, Belgium

Full list of author information is available at the end of the article
}

reported to be less sensitive to ionizing radiation since the production of free radicals, which are responsible for lethal DNA breaks, increases with the $\mathrm{O}_{2}$ partial pressure $\left(\mathrm{pO}_{2}\right)$ [16-18].

Internal radiotherapy differs from EBRT by higher tumor-absorbed doses delivered at a lower dose rate and with a more heterogeneous pattern. To the best of our knowledge, the impact of hypoxia on tumor response in patients treated by internal radiotherapy has never been directly addressed. Only the effect of Hb level on global symptoms, i.e., pain or cumulative survival, was reported in the treatment for painful osseous metastases in prostate cancer with ${ }^{186}$ Re-hydroxyethylidene-diphosphonate and ${ }^{89} \mathrm{Sr}[19,20]$. The demonstration of a significant impact of anemia on tumor response in internal radiotherapy should 
allow improvements by considering strategies under intensive development in EBRT [21-26] but, as of today, totally ignored in internal radiotherapy.

Selective internal radiation therapy (SIRT) using ${ }^{90} \mathrm{Y}$ labeled microspheres is a rapidly emerging treatment of unresectable chemorefractory primary liver tumors and hepatic metastases mainly originating from colorectal cancer (CRC). Development of ${ }^{90} \mathrm{Y}$ imaging by PET [27] after the SIRT procedure, and recently by pinhole bremsstrahlung SPECT [28], proved that tumor dosimetry is feasible [29-31] and evidenced a promising relationship between absorbed dose and early metabolic response [32] as already observed using ${ }^{99 \mathrm{~m}}$ Tc-MAA-based dosimetry [33]. The aim of this study is to further analyze this relationship and to investigate whether the $\mathrm{Hb}$ level measured on the day of the SIRT procedure has an impact on the early tumor response. For this purpose, an estimate of the tumor cell survival fraction was fitted using a tumor radiosensitivity modulated by a hemoglobin enhancement factor (HEF). This radiosensitivity modulation was implemented in the similar way that was performed for the tissue oxygenation when this parameter can be directly assessed [16-18].

\section{Methods}

\section{Patient series}

Eight patients with fludeoxyglucose (FDG)-positive hepatic metastases (six from CRC and two from melanomas) underwent a $45-\mathrm{min}{ }^{90} \mathrm{Y}$ time-of-flight positron emission tomography $\left({ }^{90} \mathrm{Y}\right.$ TOF-PET) scan (Gemini TF, Philips Medical Systems, Cleveland, OH, USA) within $4 \mathrm{~h}$ following the SIRT procedure. SIRT was performed according to the standard recommendations [34] (mean activity $\pm \mathrm{SD}=$ $1.45 \pm 0.45 \mathrm{GBq})$. Two $\left[{ }^{18} \mathrm{~F}\right] \mathrm{FDG}$ PET scans were performed: a baseline (FDG-BL) scan $2.4 \pm 2.1$ weeks (mean \pm SD) before SIRT and a follow-up (FDG-FU) scan $6.8 \pm 0.8$ weeks (mean \pm SD) after SIRT (see Table 1). All scans were reconstructed using the line of response reconstruction algorithm from Philips Medical Systems (4 iterations $\times 10$ subsets). In all patients, a blood analysis including the $\mathrm{Hb}$ level was performed on the day of SIRT. All these procedures are part of the standard therapy as routinely performed in our institution. After approval by the local ethics committee, the metabolic change of 25 tumors before and after therapy was retrospectively analyzed.

\section{Delineation of volumes of interests}

A total of 25 metastatic sites were identified on the FDG-BL scan (number of sites per patient ranging from one to eight, mean $=3.1$ ). Since the purpose was to study the early response to the absorbed dose $(D)$, viable areas of large, partly necrotic tumors were delineated using the FDG-BL scan and were analyzed as individual sites with their own absorbed dose, while the necrotic area was not incorporated in the analysis. Volumes of interest (VOIs) were manually drawn on the fused FDG-BL and ${ }^{90}$ Y-PET images. The same VOIs were used for the evaluation of the FDG-FU scan. Some undertreated viable metastatic sites, i.e., visible on the FDG-BL scan but not well targeted by the microspheres, disclosed a large size increase between the FDG-BL and FDG-FU scans (see Figure 1). In this case, the size of the VOI was then tuned accordingly in order to encompass all the metabolic cells of the metastatic site that were visible on the FDG-FU scan, i.e., the survival cells and the new cells originating from the site regrowing. A VOI of about $100 \mathrm{ml}$ was drawn in a region of the liver that was considered healthy on the basis of the FDG scans, CT scans, and MRI.

\section{Calculation of absorbed dose}

The dosimetry was obtained using a previously validated method [30]: the ${ }^{90}$ Y-PET images were 3D, convolved with a dose deposition kernel directly providing the voxel-based dosimetry (in milligray per megabecquerel). Individual tumor-absorbed dose (in gray) was determined as the mean of the voxel-based dosimetry in the ${ }^{90}$ Y-PET VOI times the total injected activity.

\section{Determination of the early response}

The tumor metabolic ratio at follow-up (MR) was calculated as:

$$
\mathrm{MR}=\frac{\left(\mathrm{SUV}_{\mathrm{FU}}^{\mathrm{tum}}-S U V_{\mathrm{FU}}^{\mathrm{liv}}\right) \times \mathrm{Vol}_{\mathrm{FU}}^{\mathrm{tum}}}{\left(S U V_{\mathrm{BL}}^{\mathrm{tum}}-S U V_{\mathrm{BL}}^{\mathrm{liv}}\right) \times \mathrm{Vol}_{\mathrm{BL}}^{\mathrm{tum}}} \times \frac{S U V_{\mathrm{BL}}^{\mathrm{liv}}}{S U V_{\mathrm{FU}}^{\mathrm{liv}}},
$$

where SUV is the mean FDG standardized uptake value, and Vol is the volume of the VOI. Assuming that the metabolism of the tumor cells does not change too much between the FDG-BL and FDG-FU scans, the mean tumor SUV multiplied by the tumor volume is related to the number of living cells in the tumor. Two improvements were introduced in Equation 1: (1) subtraction of the mean liver SUV that takes into account the fact that, due to the replacement by normal liver cells and to the finite spatial resolution of the PET system, the measured mean SUV of a responding tumor may not be different than that of the liver; and (2) multiplication by the mean liver SUV inverse ratio that corrects, to some extent, for the variation of the patient glycemia between the FDG-BL and FDG-FU scans and for a possible metabolic boosting resulting from a general inflammation of the liver and hepatic metastasis as an effect of irradiation. Different variations of this tumor-to-liver ratio method were already used by others [35-37]. 
Table 1 Patients' dosimetric data

\begin{tabular}{|c|c|c|c|c|c|c|c|c|}
\hline Patient & Type & $\begin{array}{l}\text { Site } \\
\text { number }\end{array}$ & $\begin{array}{l}\mathrm{Hb} \\
(\mathrm{g} / \mathrm{dL})\end{array}$ & $\begin{array}{l}\text { BL time } \\
\text { (weeks) }\end{array}$ & $\begin{array}{l}\text { FU time } \\
\text { (weeks) }\end{array}$ & MR & $D$ (Gy) & $\begin{array}{l}\text { Volume BL } \\
\text { (ml) }\end{array}$ \\
\hline \multirow[t]{2}{*}{1} & mel. & 1 & 10.0 & -0.2 & 6.1 & 0.74 & $233.2 \pm 52.1$ & 11 \\
\hline & & 2 & & & & 1.14 & $130.0 \pm 42.3$ & 31 \\
\hline \multirow[t]{2}{*}{2} & CRC & 1 & 13.4 & -6.2 & 8.1 & 0.11 & $169.9 \pm 79.9$ & 58 \\
\hline & & 2 & & & & 4.00 & $6.1 \pm 8.2$ & 17 \\
\hline 3 & CRC & 1 & 11.1 & -3.2 & 7.2 & 0.18 & $172.2 \pm 55.8$ & 127 \\
\hline \multirow[t]{8}{*}{4} & CRC & 1 & 14.8 & -2.3 & 6.2 & 0.15 & $110.9 \pm 33.1$ & 93 \\
\hline & & 2 & & & & 6.25 & $52.1 \pm 18.5$ & 34 \\
\hline & & 3 & & & & 19.52 & $5.0 \pm 10.8$ & 11 \\
\hline & & 4 & & & & 20.20 & $6.4 \pm 12.4$ & 25 \\
\hline & & 5 & & & & 1.67 & $18.5 \pm 17.0$ & 32 \\
\hline & & 6 & & & & 1.43 & $8.4 \pm 11.6$ & 37 \\
\hline & & 7 & & & & 3.33 & $7.9 \pm 7.6$ & 6 \\
\hline & & 8 & & & & 0.10 & $131.0 \pm 29.8$ & 10 \\
\hline \multirow[t]{2}{*}{5} & CRC & 1 & 14.3 & -3.5 & 7.2 & 0.05 & $178.5 \pm 35.2$ & 34 \\
\hline & & 2 & & & & 1.75 & $20.4 \pm 6.1$ & 6 \\
\hline \multirow[t]{6}{*}{6} & CRC & 1 & 13.0 & -2.9 & 6.1 & 0.32 & $113.9 \pm 21.9$ & 3 \\
\hline & & 2 & & & & 0.34 & $114.7 \pm 14.8$ & 3 \\
\hline & & 3 & & & & 0.22 & $127.3 \pm 24.8$ & 4 \\
\hline & & 4 & & & & 49.32 & $14.6 \pm 2.8$ & 5 \\
\hline & & 5 & & & & 0.86 & $52.0 \pm 9.6$ & 3 \\
\hline & & 6 & & & & 0.89 & $55.5 \pm 10.7$ & 4 \\
\hline \multirow[t]{2}{*}{7} & CRC & 1 & 13.1 & -0.6 & 6.1 & 1.32 & $52.2 \pm 10.6$ & 37 \\
\hline & & 2 & & & & 2.08 & $26.6 \pm 6.2$ & 36 \\
\hline \multirow[t]{2}{*}{8} & mel. & 1 & 13.4 & -0.2 & 7.0 & 0.26 & $115.0 \pm 17.8$ & 7 \\
\hline & & 2 & & & & 0.17 & $147.0 \pm 21.2$ & 6 \\
\hline
\end{tabular}

BL, baseline FDG; CRC, colorectal cancer; $D$, dose; FU, follow-up FDG; Hb, hemoglobin; mel., melanoma; MR, tumor metabolic ratio.

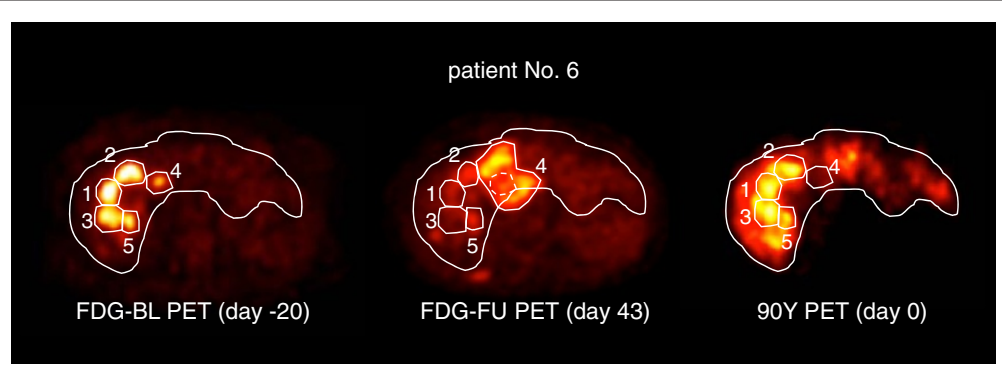

Figure 1 PET scans. One transverse slice of the patient No. 6 showing the metabolic sites of its large necrotic tumour that received different absorbed dose. The FDG uptake of the metabolic sites 1,2,3,5, that were well targeted by the microspheres, decreased between the FDG-BL and FDG-FU scans, while the metabolic site 4 not targeted by the microspheres rapidly increased in FDG uptake and in size as well, to become a new large necrotic metastatic site. The VOI on the FDG-FU scan was increased to encompass all the FDG activity originating from the increased site 4. 


\section{Early dose-response analysis}

The early dose-response relationship was fitted by minimizing:

$$
\chi^{2}=\Sigma p, m\left|S F_{p, m}-S F_{p, m}^{\bmod }\right|^{2}
$$

where $m$ is the index of the metastatic site in patient $p$, and $\mathrm{SF}$ is the survival fraction, i.e., the ratio between the number of living cells just after and before the therapy. SF is related to the metabolic ratio measured at follow-up by:

$$
S F_{p, m}=e^{-\ln (2) \Delta t_{p} / D T} M R_{p, m},
$$

where $\Delta t_{p}$ is the delay between the FDG-FU and FDG-BL scans, and DT is the tumor doubling time. The modeled survival fraction is:

$$
S F_{p, m}^{\bmod }=e^{-\alpha H E F_{p} D_{p, m}},
$$

where $\alpha$ expresses the ability of one particle to induce a DNA double-strand break. HEF was assumed to be the same for all metastatic sites $m$ in patient $p$ and related to the $\mathrm{Hb}$ level (in gram per deciliter) according to:

$$
H E F_{p}=1+k\left(H b_{p}-13\right) .
$$

This results in a global fit of three parameters, DT (days), $\alpha$ (per gray), and $k$ (deciliter per gram), on 25 points (number of metastatic sites), where DT and $\alpha$ were constrained to be positive.

HEF was introduced in Equation 4 in the similar way that the oxygen enhancement ratio (OER) was introduced for acute hypoxia $[17,18]$. Equation 5 was designed to give $\mathrm{HEF}=1$ for patients having a $\mathrm{Hb}$ level of $13 \mathrm{~g} / \mathrm{dL}$, which is a normal value. This is an arbitrary choice; a value other than $13 \mathrm{~g} / \mathrm{dL}$ will just give other values for $\alpha$ and $k$ but will give an identical fit quality.

\section{Results and discussion Results}

Figure 2 shows the relationship between the absorbed doses and the response, estimated as the tumor metabolic ratio at approximately 7 weeks post-therapy for all metastatic sites. The tumor response was highly variable between patients but, per patient, was clearly dependent on the absorbed dose. Two patients showed a convincing response (gray squares and brown circle), one patient had two nonresponding tumors (cyan hexagons), whereas the remaining patients displayed a mix of responding and nonresponding tumors, depending on the absorbed doses.

All tumors show a trend towards a similar proliferation rate by extrapolation of the response for $D=0$, except for four metastases that clearly behave as outliers (Figure 2, see box in the left upper corner). These four metastases belong to two patients with a long disease and therapy history ( 2 and 4 years). It is likely that these metastases may have acquired a phenotype resulting in a doubling time significantly shorter than the mean of the other metastases. The global fit is performed assuming that all the metastases have the same doubling time (DT, Equation 3); accordingly, these four metastases were considered to be outside the application domain of the model and were ruled out for the global fit.

The global fit, run to estimate the biological parameters, was therefore performed on the 21 remaining metastases using SigmaPlot 2000 version 6.00 (Systat Software Inc, San Jose, CA, USA) and resulted in the following values (best parameter $= \pm 2.1^{*}$ std error, i.e., $95 \%$ confidence interval): DT $=50 \pm 8$ day, $\alpha=0.018 \pm 0.003$ / Gy and $k=0.23 \pm 0.05 \mathrm{dL} / \mathrm{g}$. The left axis in Figure 3 shows the tumor cell survival fraction (Equation 3 ) as a function of $\operatorname{HEF}_{p} D_{p, m}$ that takes into account the $\mathrm{Hb}$ level (Equation 5). The line is the survival fraction modeled by Equation 4 using the parameters determined by the global fit $(R=0.96)$. It can be observed that a number of tumors showed a metabolic ratio higher than one (right axis) even though their survival fraction was lower than one. These tumors actually showed a response that is not sufficient to counterbalance at week 7 the regrowth of the viable tumor cells.

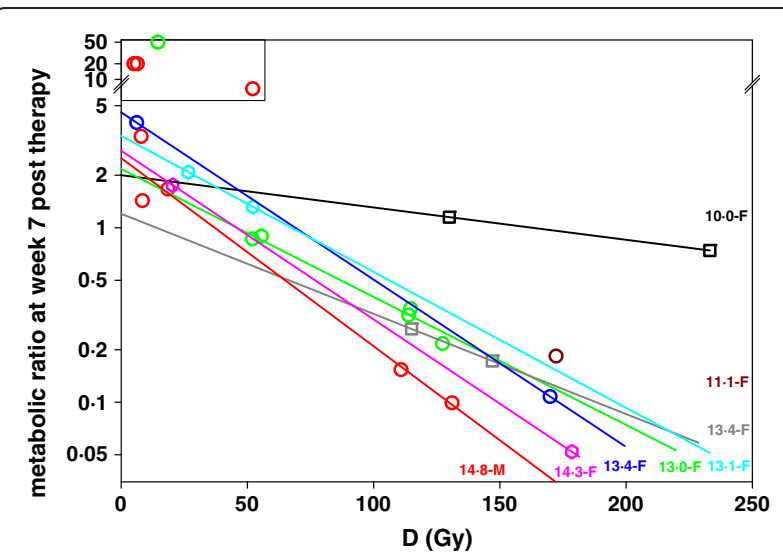

Figure 2 Relationship between absorbed doses D and tumor metabolic ratio $(M R)$. MR for the responding tumors are lower than 1. Each color refers to each patient (black - 1, blue - 2, brown - 3, red 4, pink - 5, green - 6, cyan - 7, and grey - 8). Open circles and hexagons correspond to metastases from CRC, and open squares refer to metastases from melanoma. The patient gender and the $\mathrm{Hb}$ level (in gram per deciliter) on the day of the SIRT procedure are indicated at the extremity of each fitted line. Individual patient tumors were fitted by $g f e^{-\bar{a} D}$, where $g f$ is the growing fraction in the absence of an absorbed dose and $\bar{a}$ is the overall cell radiosensitivity, i.e., containing the HEF factor. The box in the upper left corner identifies outliers (see 'Results and discussion'). The lines are the patient data individually fitted with this single exponential. 


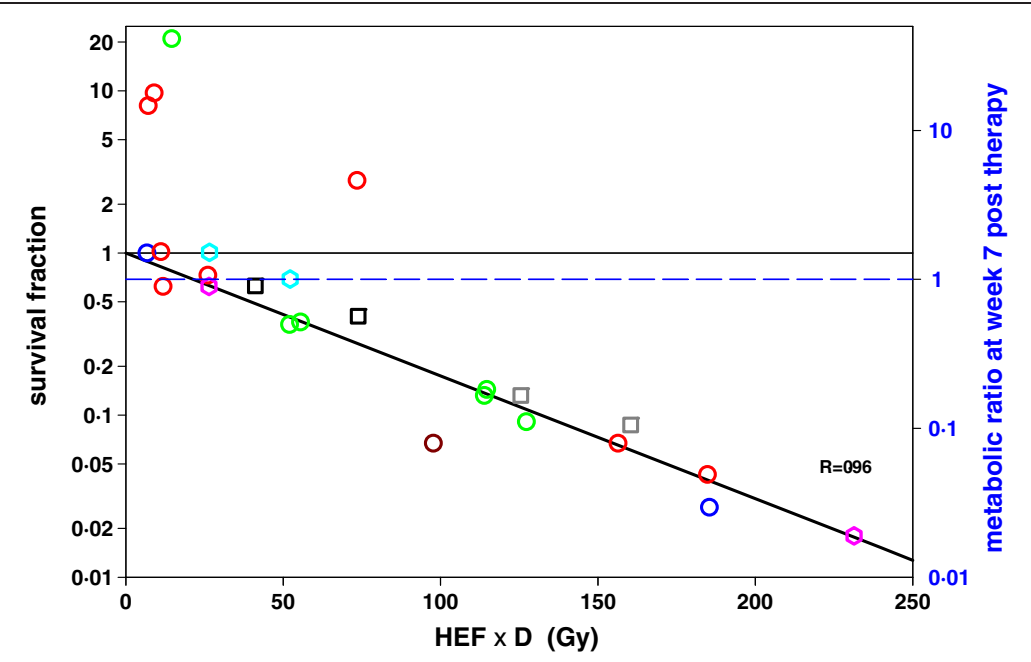

Figure 3 Tumors survival fraction. Relationship between absorbed dose D corrected by the HEF and the tumor cell survival fraction (left axis) and metabolic ratio (right axis) calculated at week 7 post-therapy using the doubling time obtained by the global fit. For colors and symbols refer to Figure 2. The line corresponds to Equation 4 with the parameters $a, k$ and DT minimizing Equation 2, excluding the four metastases with SF $>1$, which proved their shorter DT (using the actual metastasis DT in Equation 3 should necessarily give SF $\leq 1$ ). These four metastases were already considered as outliers from Figure 2. See animation of the fit in Additional file 1.

The optimal values of HEF for each patient in relation with their $\mathrm{Hb}$ level are shown in Figure 4. This graphical representation of Equation 5 indicates that the prediction of response in individual patients, taking into account their $\mathrm{Hb}$ level, is very close $(R=0.94)$ to what would be expected from the global fit (see Additional file 1).

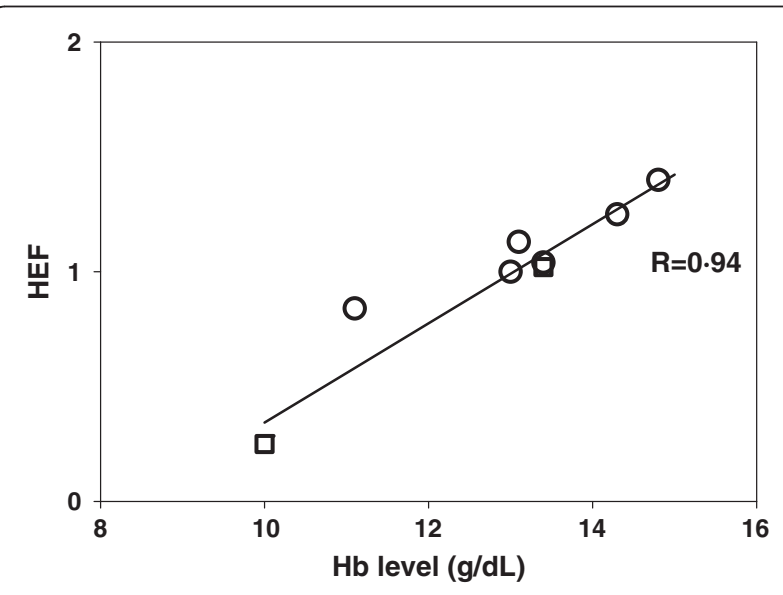

Figure 4 Optimal values of HEF for each patient in relation with their $\mathrm{Hb}$ level. Relationship between the $\mathrm{Hb}$ level and the optimal values of the $\mathrm{HEF}_{p}$ parameter obtained by individually fitting HEF for each patient dose-response using $a$ and DT values determined from the global fit. The line corresponds to Equation 5, with $k$ obtained by the global fit. $R$ is the correlation coefficient obtained by making a linear regression of these optimal $\mathrm{HEF}_{p}$.

\section{Discussion}

This study clearly shows the impact of $\mathrm{Hb}$ level on tumor response. This impact was quantitatively interpreted, introducing a HEF, as the effect of tumor hypoxia induced by the patients' anemia (Figure 3). This is the first time that such analysis was applied to in vivo human data. This methodology is usually limited to preclinical models, such as cell assays or xenograft tumors in rodents. In preclinical models, almost all parameters are under control, such as the tumor cell line and time of progression. In the clinics, there is a large heterogeneity of metastases between patients but also within the same patient. In particular, the duration of progression of a single lesion in a particular patient is usually totally overlooked. This may lead to variations in the tumor cell phenotype, with some metastases becoming significantly more proliferative. We initially assessed tumor metabolic response using FDG uptake without knowledge of any other parameters. As already observed using morphological estimates, 50 Gy was required to observe any response. There was a clear trend towards a dose-response relationship, but the correlation was weak $(R=0.51)$. After observing the different response patterns between patients, we looked at the $\mathrm{Hb}$ level as a possible factor accounting for this variance. After individually fitting patient data and highlighting the $\mathrm{Hb}$ level in Figure 2, it appeared obvious that some relationship did exist. We therefore applied the concept of a radiosensitivity modulation to the data, a paradigm that is classically utilized for in vitro data, and that, to the best of our knowledge, 
has never been applied to internal radiotherapy of patients.

This correction factor appeared tightly related to the $\mathrm{Hb}$ level and, hence, was called HEF. For Hb level ranging between 10 and $15 \mathrm{~g} / \mathrm{dL}$, the individual HEF estimate displayed a linear increase, reaching a ratio of 5.6 between these two extreme $\mathrm{Hb}$ levels (Figure 4). This is in keeping with the major impact of $\mathrm{Hb}$ level observed for EBRT in recurrent rectal cancer [38]. This is also in line with the observation of Vaupel et al. [9] showing by an invasive method that the tumor $\mathrm{pO}_{2}$ can vary by a factor 4 in breast cancer as a function of the $\mathrm{Hb}$ level. Such linear relationship is however not the rule: in uterine and cervical cancer and in head and neck cancer, a bell-shaped relationship was reported by Vaupel et al. [9], with a maximal $\mathrm{pO}_{2}$ value measured for $\mathrm{Hb}$ levels of 12.5 and $14 \mathrm{~g} / \mathrm{dL}$, respectively. Such intraoperative $\mathrm{pO}_{2}$ measurements are lacking for hepatic metastases from CRC.

The radiation sensitivity parameter alpha found (0.018/Gy for a normal $\mathrm{Hb}$ level of $13 \mathrm{~g} / \mathrm{dL})$ is about 15 times lower than values found in cell assays [39]: 0.31/ Gy for melanoma and $0.25 /$ Gy for CRC. However, this is consistent with the comparison of in vivo xenograft and in vitro data that suggests that the survival is considerably higher in vivo [40]. In a retrospective analysis of SIRT in 73 patients with hepatocellular carcinoma, Strigari et al., using a radiobiological model of tumor control probability (TCP) assessed in CT scan by the RECIST criteria but without addressing anemia, found two very low extreme bounds for the value of alpha [41]: 0.001 and $0.005 / G y$, much lower than values reported using cell assays [42]: 0.1 and $0.4 / \mathrm{Gy}$ for HepG2 and Hep3b cell lines, respectively. The factor 5 between the extreme bounds of the radiosensitivity found by Strigari et al. is similar to the factor 5.6 that we found between patients with extreme Hb levels.

Kato et al. [43] set up an elegant experiment that enlightens the observations listed in both previous paragraphs. They modeled chronic hypoxia, which is usually the rule in patients, by culturing gastric cancer cells (OCUM-12 line) in successively decreasing partial oxygen pressures (pO2). An important fraction of cells died, but the survivors resulted in an OCUM-12 line that was still able to proliferate at $13 \mathrm{mmHg}$ pO2 (called OCUM-12/hypo). The fit of the survival curves they measured shows that the chronic $13 \mathrm{mmHg}$ pO2 exposure reduced by a factor 14 the radiosensitivity $\alpha$ of the OCUM-12/hypo line as compared to that of the original OCUM-12 parent line set in acute $13 \mathrm{mmHg}$ pO2 for $24 \mathrm{~h}$. For comparison, acute $13 \mathrm{mmHg}$ pO2 in most cell lines decreases the radiosensitivity by only a factor of about 1.2 versus normoxic conditions ([44], Figure 3). Lastly, the results of Vaupel et al. [9] show that, for a normal $\mathrm{Hb}$ level of $13 \mathrm{~g} / \mathrm{dL}$, the typical tumor pO2 is in fact about $13 \mathrm{mmHg}$ due to the disturbed vascular architecture.

In a previous study on ${ }^{90} \mathrm{Y}$-DOTATOC therapy in patients with neuroendocrine tumors [45], we have shown for the first time a good relationship between tumor shrinkage and absorbed dose using morphological imaging. There was, however, considerable spread in this dose-response relationship. In the current study, the correlation is excellent $(R=0.96)$ and with little spread. This can be explained by the presence of more favorable features in the current study: (1) the absorbed dose is directly assessed from the distribution of the therapeutic agent, (2) the effective half-life is assumed identical to that of the physical decay and thus known with high accuracy, and (3) the Hb enhancement effect was taken into consideration.

In this study, the mean absorbed dose modulated by the $\mathrm{Hb}$ level proved being sufficient to correlate to the tumor metabolic response. Using this mean absorbed dose, the cell killing is overestimated in the regions of the VOI that received lower dose, but is underestimated elsewhere. Both effects tend to average. Further refinement to account for the dose distribution in the VOI, such as using the equivalent uniform dose (EUD), will also require the assessment of living cell distribution in this VOI. This could be done using the base-line FDG scan. However, due to tumor evolution, the delay in our study between the baseline FDG and the ${ }^{90} \mathrm{Y}$-microsphere scans makes a tumor co-registration on a voxel basis between the two scans impossible.

Conceptually, the doubling time could be assessed by extrapolating for each patient the absorbed dose-metabolic ratio relationship to the left axis (i.e., $D=0$ ), as shown in Figure 2. However, this method is not applicable in view of the following: (1) the doubling time is not only patient dependent but also metastasis dependent (illustrated by the red and green circles), and (2) some patients have only one tumor (brown circle) or only two metastases far away from the left axis (gray squares), making the extrapolation impossible or very unstable, respectively. Accordingly, a single doubling time value common to all the metastasis was used in the global fit, and the value found (50 days) is in good agreement with CEA doubling time measured in 33 patients with hepatic CRC metastases ( $47 \pm 25$ days) [46]. Individually fitting the four outlier metastases using Equations 3 and 4 with $\alpha=0$ allows giving an upper bound for their doubling time that was found to be $22.5,13.9,13.7$ and 11.2 days, respectively. In a prospective clinical study, the individual metastasis doubling time could be assessed by acquiring an additional FDG scan close to the ${ }^{90}$ Y-PET scan, the FDG-BL scan being usually performed a few weeks before the SIRT 
procedure. This procedure should also help to investigate whether a lag time before regrowth starts after irradiation should be introduced in Equation 3, such as proposed in other models [47].

Our results showed that a higher $\mathrm{Hb}$ level is important to get an optimal tumor response in ${ }^{90} Y$ SIRT. Various correction methods to overcome tumor hypoxia in EBRT are under clinical investigation and intensively reviewed [21-26]: red cell transfusion or the use of recombinant human erythropoietin to correct anemia, enriched oxygen breathing or hyperbaric condition to increase tumor oxygenation, and the use of radiosensitizing drugs. Although some strategies are currently being proposed, contradictory results still exist between clinical studies. These studies are based on the late assessment of the disease outcome: local tumor control using morphological measurements (e.g., CT scan) or patient cumulative survival curves. Of course, these clinical studies are highly demanded because they really assess the final purpose of the therapy. However, they require large patient series and long follow-up period. Up to now, the quantitative analysis of the relationship between SF and absorbed dose has been widely performed on in vitro assays. Similar studies in rodent models allow obtaining this information on an individual tumor cell line in a small animal series. The same paradigm applied here allows, in individual patient, assessment of the effect of hypoxia on several tumors. This may, in turn, be used to evaluate potential optimization protocols in small patient series.

\section{Conclusions}

The current results show that absorbed dose alone is not sufficient to predict early tumor response, but this become feasible when introducing a simple hemoglobin enhancement factor linear to the patient's $\mathrm{Hb}$ level. While the oxygen effect is an important field of research in EBRT, these results support the study of similar strategies to reduce hypoxia in the framework of internal radiotherapy. The quantitative analysis of the relationship between tumorabsorbed doses and early response has the power to allow fast screening of hypoxia correction methods in limited patient series. Internal radiotherapy may be more efficient if performed earlier in the therapy line, when anemia related to disease progression or to treatment remains limited.

\section{Additional file}

Additional file 1: Hemoglobin level significantly impacts the tumor cel survival fraction in humans after internal radiotherapy: application of a preclinical radiobiology model to clinical data.

Competing interests

The authors declare that they have no competing interests.
Acknowledgments

We thank Thomas Paulus for his expert technical assistance in performing the ${ }^{90} Y$ TOF-PET.

\section{Author details}

'Department of Nuclear Medicine, Cliniques Universitaires Saint Luc, Université Catholique de Louvain, Avenue Hippocrate 10, Brussels 1200, Belgium. ${ }^{2}$ Department of Interventional Radiology, Cliniques Universitaires Saint Luc, Université Catholique de Louvain, Avenue Hippocrate 10, Brussels 1200, Belgium. ${ }^{3}$ Department of Oncology, Cliniques Universitaires Saint Luc, Université Catholique de Louvain, Avenue Hippocrate 10, Brussels 1200, Belgium.

\section{Authors' contribution}

SW elaborated the Hb-dependent dosimetry model and performed the results analysis. RL and FJ performed the study design. RL, PG, and MVE carried out the treatment and follow-up of the patients. SW, RL, SP, and FJ participated in the paper writing. All authors read and approved the final manuscript.

Received: 9 February 2012 Accepted: 19 May 2012

Published: 19 May 2012

\section{References}

1. Caro JJ, Salas M, Ward A, Goss G: Anemia as an independent prognostic factor for survival in patients with cancer. A systemic quantitative review. Cancer 2001, 91:2214-2221.

2. Harrison LB, Shasha D, Homel P: Prevalence of anemia in cancer patients undergoing radiotherapy: prognostic significance and treatment. Oncology 2002, 63:11-18

3. Harrison LB, Chadha M, Hill RJ, Hu K, Shasha D: Impact of tumour hypoxia and anemia on radiation therapy outcomes. Oncologist 2002, 7:492-508.

4. Nowrousian MR: Prevalence, pathophysiology, predictive factors, and prognostic significance of anemia in cancer chemotherapy. In Recombinant human erythropoietin (rhEPO) in clinical oncology. 2nd edition. Edited by Nowrousian MR. Wien, New York: Springer; 2002:63-100.

5. Van Belle SJ-P, Cocquyt V: Impact of haemoglobin levels on the outcome of cancers treated with chemotherapy. Crit Rev Oncol Hematol 2003, 47:1-11.

6. Clarke H, Pallister CJ: The impact of anaemia on outcome in cancer. Clin Lab Haem 2005, 27:1-13.

7. Österborg A: Anaemia in patients with cancer: association to prognosis and prediction of response to erythropoietic agents. In Anaemia in Cancer. Edited by Bokemeyer C, Ludwig H. Edinburgh London New York: Elsevier; 2005.

8. Vaupel $P$, Mayer A: Hypoxia in cancer: significance and impact on clinical outcome. Cancer Metastasis Rev 2007, 26(2):225-239.

9. Vaupel P, Mayer A, Höckel M: Relationship between hemoglobin levels and tumour oxygenation. In Recombinant human erythropoietin (rhEPO) in clinical oncology. Edited by Nowrousian MR. Wien, New York: Springer; 2008.

10. Höckel M, Vaupel P: Tumour hypoxia: definitions and current clinical biologic, and molecular aspects. J Natl Cancer Inst 2001, 93:266-276.

11. Semenza GL: Hypoxia, clonal selection, and the role of HIF-1 in tumour progression. Crit Rev Biochem Mol Biol 2000, 35:71-103.

12. Semenza GL: Involvement of hypoxia-inducible factor 1 in human cancer. Intern Med 2002, 41:79-83.

13. Semenza GL: HIF-1 and tumour progression: pathophysiology and therapeutics. Trends Mol Med 2002, 8(suppl 1):S62-S67.

14. Harris AL: Hypoxia-a key regulatory factor in tumour growth. Nat Rev Cancer 2002, 2:38-47.

15. Leo C, Giaccia AJ, Denko NC: The hypoxic tumour microenvironment and gene expression. Semin Radiat Oncol 2004, 14:207-214.

16. Stabin MG: Fundamentals of nuclear medicine dosimetry. New York: Springer; 2008.

17. Beyzadeoglu M, Ozyigit G, Ebruli C: Radiobiology. In Basic radiation oncology. Edited by. New York: Springer; 2010.

18. McParland BJ: Biological effects of ionizing radiation. In Nuclear Medicine Radiation Dosimetry. Edited by. New York: Springer; 2010.

19. van der Poel HG, Antonini N, Hoefnagel CA, Horenblas S, Valdes Olmos RA Serum hemoglobin levels predict response to strontium- 89 and rhenium-186-HEDP radionuclide treatment for painful osseous metastases in prostate cancer. Urol Int 2006, 77(1):50-56. 
20. Windsor PM: Predictors of response to strontium-89 (Metastron) in skeletal metastases from prostate cancer: report of a single centre's 10-year experience: palliation and metastatic disease. Clin Oncol 2001, 13(3):219-227.

21. Wardman P: Chemical radiosensitizers for use in radiotherapy. Clin Oncol 2007, 19(6):397-417.

22. Ahn GO, Brown JM: Combinations of hypoxia-targeting compounds and radiation-activated prodrugs with ionizing radiation. In Multimodal concepts for integration of cytotoxic drugs and radiation therapy. Edited by. New York: Springer; 2006:67-91 [Brown MJ, Nieder C, Brady LW (Series Editors): Medical Radiology].

23. Janssen HL, Haustermans KM, Balm AJ, Begg AC: Hypoxia in head and neck cancer: how much, how important? Head Neck 2005, 27(7):622-638.

24. Hu K, Harrison LB: Impact of anemia in patients with head and neck cance treated with radiation therapy. Curr Treat Options Oncol 2005, 6(1):31-45.

25. Ozsahin M, Azria D, Beer K, Mirimanoff RO, Zouhair A: External radiotherapy and anaemia treatment: state of the art. Swiss Med Wkly 2005, 135(1-2):4-10

26. Horsman MR, van der Kogel AJ: Therapeutic approaches to tumour hypoxia. In Basic clinical radiobiology. Edited by Joiner M, van der Kogel A. London: MPG Books; 2009.

27. Lhommel R, Goffette P, Van den Eynde M, Jamar F, Pauwels S, Bilbao J, Walrand S: Yttrium-90 TOF PET scan demonstrates high-resolution biodistribution after liver SIRT. Eur J Nucl Med Mol Imaging 2009, 36(10):1969.

28. Walrand S, Hesse S, Demonceau G, Pauwels S, Jamar F: Yttrium-90 labeled microspheres tracking during liver selective internal radiotherapy by bremsstrahlung pinhole SPECT: feasibility study and evaluation in an abdominal phantom. EJNMMI Res 2011, 1:32

29. Werner MK, Brechtel K, Beyer T, Dittmann H, Pfannenberg C, Kupferschläger $\mathrm{J}$ : PET/CT for the assessment and quantification of ${ }^{90} \mathrm{Y}$ biodistribution after selective internal radiotherapy (SIRT) of liver metastases. Eur J NuCl Med Mol Imaging 2010, 37(2):407-408.

30. Lhommel R, van Elmbt L, Goffette P, Van den Eynde M, Jamar F, Pauwels S, Walrand S: Feasibility of yttrium-90 TOF-PET based dosimetry in liver metastasis therapy using SIR-spheres. Eur J Nucl Med Mol Imaging 2010, 37 (9):1654-1662.

31. van Elmbt L, Vandenberghe S, Walrand S, Pauwels S, Jamar F: Comparison of yttrium-90 quantitative imaging by TOF and non-TOF PET in a phantom of liver selective internal radiotherapy. Phys Med Biol 2011, 56 (21):6759-6777.

32. Walrand S, Flux GD, Konijnenberg MW, Valkema R, Krenning EP, Lhommel R, Pauwels S, Jamar F: Dosimetry of yttrium-labelled radiopharmaceuticals for internal therapy: (86)Y or (90)Y imaging? Eur J Nucl Med Mol Imaging 2011, 38(1 suppl 1):S57-S68.

33. Garin $E$, Lenoir $L$, Rolland $Y$, Edeline J, Mesbah $H$, Laffont $S$, Porée $P$, Clément B, Raoul JL, Boucher E: Dosimetry based on 99mTc-macroaggregated albumin SPECT/CT accurately predicts tumor response and survival in hepatocellular carcinoma patients treated with 90Y-loaded glass microspheres: preliminary results. J NuCl Med 2012, 53(2):255-263.

34. Kennedy A, Nag S, Salem R, Murthy R, McEwan AJ, Nutting C, Benson A 3rd, Benson A, Espat J, Bilbao JI, Sharma RA, Thomas JP, Coldwell D: Recommendations for radioembolization of hepatic malignancies using yttrium-90 microsphere brachytherapy: a consensus panel report from the radioembolization brachytherapy oncology consortium. Int J Radiat Oncol Biol Phys 2007, 68(1):13-23.

35. Findlay $M$, Young $H$, Cunningham D, Iveson A, Cronin B, Hickish T, Pratt B, Husband J, Flower M, Ott R: Noninvasive monitoring of tumour metabolism using fluorodeoxyglucose and positron emission tomography in colorectal cancer liver metastases: correlation with tumour response to fluorouracil. J Clin Oncol 1996, 14:700-708.

36. Flamen $P$, Van Cutsem E, Lerut A, Cambier JP, Haustermans K, Bormans G, De Leyn P, Van Raemdonck D, De Wever W, Ectors N, Maes A, Mortelmans L: Positron emission tomography for assessment of the response to induction radiochemotherapy in locally advanced oesophageal cancer. Ann Oncol 2002, 13(3):361-368.

37. Mackie GC, Shulkin BL, Ribeiro RC, Worden FP, Gauger PG, Mody RJ, Connolly LP, Kunter G, Rodriguez-Galindo C, Wallis JW, Hurwitz CA, Schteingart DE: Use of [18F]fluorodeoxyglucose positron emission tomography in evaluating locally recurrent and metastatic adrenocortical carcinoma. J Clin Endocrinol Metab 2006, 91(7):2665-2671.

38. Rades D, Kuhn H, Schultze J, Homann N, Brandenburg B, Schulte R, Krull A, Schild SE, Dunst J: Prognostic factors affecting locally recurrent rectal cancer and clinical significance of hemoglobin. Int J Radiat Oncol Biol Phys 2008, 70(4):1087-1093.

39. Fertil B, Malaise EP: Intrinsic radiosensitivity of human cell lines is correlated with radioresponsiveness of human tumours: analysis of 101 published survival curves. Int J Radiat Oncol Biol Phys 1985, 11(9):1699-1707.

40. Malaise EP, Fertil B, Chavaudra N, Guichard M: Distribution of radiation sensitivities for human tumour cells of specific histological types: comparison of in vitro to in vivo data. Rad Oncology Biol Phys 1986 12:617-624.

41. Strigari L, Sciuto R, Rea S, Carpanese L, Pizzi G, Soriani A, laccarino G, Benassi $M$, Ettorre GM, Maini CL: Efficacy and toxicity related to treatment of hepatocellular carcinoma with 90Y-SIR spheres: radiobiologic considerations. J Nucl Med 2010, 51(9):1377-1385.

42. Zheng XK, Chen LH, Yan X, Wang HM: Impact of prolonged fraction dosedelivery time modeling intensity-modulated radiation therapy on hepatocellular carcinoma cell killing. World J Gastroenterol 2005, 11 (10):1452-1456.

43. Kato Y, Yashiro M, Fuyuhiro Y, Kashiwagi S, Matsuoka J, Hirakawa T, Noda S, Aomatsu N, Hasegawa T, Matsuzaki T, Sawada T, Ohira M, Hirakawa K: Effects of acute and chronic hypoxia on the radiosensitivity of gastric and esophageal cancer cells. Anticancer Res 2011, 31:3369-3376.

44. Carlson DJ, Keall PJ, Loo BW, Chen ZJ, Brown JM: Hypofractionation results in reduced tumour cell kill compared to conventional fractionation for tumours with regions of hypoxia. Int J Radiation Oncology Biol Phys 2011, 79(4):1188-1195

45. Barone R, Borson-Chazot F, Valkema R, Walrand S, Chauvin F, Gogou L, Kvols $L K$, Krenning EP, Jamar F, Pauwels S: Patient-specific dosimetry in predicting renal toxicity with (90)Y-DOTATOC: relevance of kidney volume and dose rate in finding a dose-effect relationship. J Nucl Med 2005, 46(suppl 1):99S-106S.

46. Staab HJ, Anderer FA, Hornung A, Stumpf E, Fischer R: Doubling time of circulating CEA and its relation to survival of patients with recurrent colorectal cancer. $\mathrm{Br} J$ Cancer 1982, 46(5):773-781.

47. Fowler JF: The linear-quadratic formula and progress in fractionated radiotherapy. Br J Radiol 1989, 62:679-694.

doi:10.1186/2191-219X-2-20

Cite this article as: Walrand et al:: Hemoglobin level significantly impacts the tumor cell survival fraction in humans after internal radiotherapy. EJNMMI Research 2012 2:20.

\section{Submit your manuscript to a SpringerOpen ${ }^{\circ}$ journal and benefit from:}

- Convenient online submission

- Rigorous peer review

- Immediate publication on acceptance

- Open access: articles freely available online

- High visibility within the field

- Retaining the copyright to your article

Submit your next manuscript at $>$ springeropen.com 